\title{
Performance Analysis of Energy Efficient Distributed Cluster Routing Techniques in Wireless Sensor Networks
}

\author{
Harish S.V, Archana N.V
}

\begin{abstract}
Wireless sensor network consists of small sensing nodes having unique characteristics in networks field and energy awareness routing for communication capabilities, computational power consumption. A wireless sensor network (WSN) is a grouping of sensor nodes in a network that perform to support Sensing, Signal processing, Communications and Connectivity for data processing and transmit the information to the destiny (Base station) through neighboring nodes with the help of energy source (batteries). The batteries used in WSN neither to be recharged nor be replace. It is necessary to extend the network lifetime for better performance. Many protocols have their own specific design but major issue is energy awareness. Based on number of nodes present in the field and the speeds at which the multiple parameters like Packet delivery ratio, network lifetime, overhead control are compared. In this paper, the proposed protocol is an efficient energy routing protocol which tries to provide fairness in network. Simulation results through MATLAB are presented.
\end{abstract}

Keywords: Fairness, Energy Efficiency, Cluster routing, Wireless sensor networks, mobility, Energy hole problem

\section{INTRODUCTION}

The WSN is the most popular technology in $21^{\text {st }}$ century with the development of economy and continous living standards. Consider a group of sensor network, desired to have its own network configured. To have an active node network a wake up contact has to be established so that their neighbour node to decide where to forward the collected data, at what interval of time and establish transmission power levels. It's a challenge of own routing (self-configured) protocol to gather information and transmission data for maximizing the battery levels. For setting up of a sensor networks for a uniformly distributed networks to have achieve a task lifetime maximization is a major problem. We further assume each sensor node is having finite energy consumed during active operation of the network. The traffic generated over the nodes and data aggression techniques can be applied through with uniform load within the networks. One of the most important field in wireless sensor network is coverage. It is usually defined as how long the sensor can be able to measure the quality of services (QOS). Each nodes has a different transmission range to discover its path and establish the connectivity with neighbor node. Sensors can transmit data to sink via multi-hop communication.

Revised Manuscript Received on June 15, 2020.

* Correspondence Author

Mr. Harish S.V*, Research Scholar, Dept of ECE, NIE Institute of Technology, Mysuru, Karnataka

Dr. Archana N.V, Professor and Head, Dept of EEE, NIE Institute of Technology, Mysuru Karnataka

(C) The Authors. Published by Blue Eyes Intelligence Engineering and Sciences Publication (BEIESP). This is an open access article under the CC BY-NC-ND license (http://creativecommons.org/licenses/by-nc-nd/4.0/)
The WSN make use of multihop communication for data flow transmission which may consume battery before the due lifetime. This behavior is called energy hole problem $(\mathrm{Li} \&$ Mohapatra, 2007)[1].

Multi-hop communication avails to network lifetime by load balancing in the network and consume energy across sensors. Node formation is an major issue in wsn because it has an important impact on the performance of the network. There are two types of node formation. one is randomised distribution and another is deterministic formation. Node formation technique can be used to improve the lifetime of the network. The main prime problems in WSN related to coverage are sensing range is restricted to limited radius. The main objective is to maximize the coverage area by a single sensor to the total area discovered. This consequently affects to a coverage problem.

\section{RELATED WORK}

There are different parameters to analyse in wireless sensor network performance. Among few parameters to improve network lifetime are as follows.

Network lifetime can be defined as the time at which the start of network functions to the instant at which the first node loose its energy or we call it as First death node ( FDN) in the network. One of the methodology that can be adopted is to reduce node diffusion power in order to reach its destiny through selected neighbor nodes which may save energy and also improve network throughput.

Schedule the node to be in sleep mode if the node is not in use so that the sensor will have an efficient energy.

Another method to solve energy consumption problem are based on node formation method using different protocol (algorithm) and energy consumption in different types of network should be evenly distributed. Node formation technique: To achieve the required coverage level and maximize network lifetime, one of the basic requirement is where to randomize node placement and how to schedule to bring out desired connectivity to ensure where the coverage is guarantee that every Region of interest is monitored by at least one sensor and to ensure sufficient routing paths (Moro, Gianluca and Gabreile Mont, Journal of Network and Computer application, 2012)[2]. Energy Consumption: In wireless sensor network the node that generate data is source node and the data accumulated point of node is sink (destination). The energy will consume will be more at the source node and sink node.

Published By:

Blue Eyes Intelligence Engineering

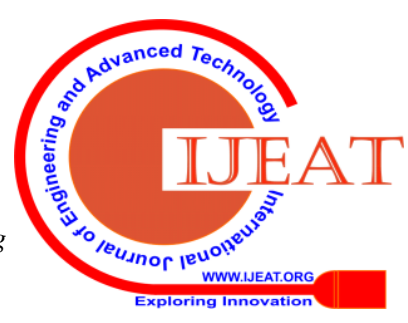


The sensor sends data to sink node through a single hop or multiple hop communication. Low Energy Adoptive Cluster Hierarchy (LEACH) protocol is cluster based protocol introduced by Heinzelman, etal, which forms a randomly distributed cluster formation [3]. A group of node connected each other form one cluster. A group of cluster becomes a cluster network. In one cluster group, one will be the cluster head and remaining will be the members of that cluster node network. Selection or identifying the cluster head among the other nodes is a major difficulty issue. Leach separates in distinct stages firstly steady state phase and another is setup phase. Steady state phase is used to transfer data to the sink node (base station). In order to reduce the overhead the duration of steady state should be greater than the setup phase [4]. In setup phase every node broadcast its priority to become a cluster head. As an elected node will become a cluster head and other nodes will be a member to that cluster. Untill the work progress complete no other node can become cluster head. Cluster head will be change for different scheduled operations.

A sensor node chooses a random number [r] between 0 and 1 . If random number is less than the threshold value [T(n)] then that node will become a cluster head for the current round (work-done).The threshold can be calculated based on desired percentage to cluster head, the current round, and the set of node that have not selected as a cluster head in last rounds.

$$
T(n)=\frac{p}{(1-p(r \bmod (1 / p))} \text { if } n \in G
$$

Where $G$ is the set of nodes that are involved in the cluster-head election. Once the cluster head is formed, Cluster head initiate the time schedule when to communicate its data from its source to destiny. This time schedule is broadcast to all the neighboring node cluster. Clustering reduce the gain of energy consumption, but protocol assumes that all node begins with uniform energy in each round and cluster head also consumes the same amount energy which may not be true. This protocol may extend to non uniform energy node and energy based threshold. Power Efficient Gathering in Sensor Information (PEGASIS) is one such routing protocol which follows chain based approach.In this protocol the sensor node organize a chain between source and destination through broadcasting a dummy packet to its neighbor and establishes a chain path to send the data from source to the sink $[5,6]$. If any node fails in between the chain is reconstructed to change its path. In order to reduce the packet loss a Leader or Cluster head will be created among the nodes, who is near to the base station (Sink node). Only leader node has a privilege to communicate with the sink node and other nodes will be a part of members of that network. This type of protocol may reduce energy consumption. Drawback of this protocol is the leader or cluster head cannot be decided how far from the base station it is located. The network delay may occur because of single leader through out the network.

DEEC is a well-known energy-efficient protocol for heterogeneous WSN's to estimate the network life time for a given metrics (Qing et al., 2006) [7]. In this protocol the sensor networks divided into different cluster, Each having one cluster head based on the probability function it selects and other nodes remains as a part of member in the network field. Cluster head collects the information and sends to the base station.

To select a cluster head in a network combination of residual energy and average energy of network is considered. In a WSN network it is assumed different amount of energy initially and newly added nodes have more energy than the previous nodes [7].

To reduce the energy consumption in the sensor network scaling factor parameter is introduced, which assume that nodes are directly connected to the base station with the network field.

$$
\begin{aligned}
& \text { Scaling factor }= \\
& \text { \{rand()X } \frac{\text { Area of network field }}{\text { Area of cluster X no.of nodes in a cluster }}
\end{aligned}
$$

For DEEC protocol, Initially define the parameter based on the network size, order of the node and scaling factor. The number of nodes and the cluster head percentage should be considered. Determine the current energy of the node and the energy of the current round. compute the average energy and residual energy of the node to become the cluster head

$$
n_{1}=\frac{I}{p_{1}}=\frac{E(r)}{P_{0_{0} E_{1}(r)}}=n_{00 r} \frac{E(r)}{E_{1}(r)}
$$

Once the $\mathrm{CH}$ is defined the neighboring nodes will be a members of the network. Compare the energy of the selected node to modify the threshold probability. If the node is greater than the threshold then node acts as a cluster. This process continues till the optimum result.

The main impact of this DEEC protocol is to

- To host a better scaling factor parameter to minimize the energy of nodes within cluster.

- To suggest a new modified Equation to compute threshold probability more resourcefully.

- To extend network life span by using neighborhood information concept.

\section{NETWORK MODEL}

Assume all the sensor nodes are randomly distributed and become stationary. Node has an information about its energy level and distance of its neighbors. The transmission of data gathering between two nodes will be established only if they are within the range of each other. A node can compute its distance based on distance of another node on the received signal strength (Bellman ford algorithm).

\section{Performance Metrics:}

Following are the performance metrics for evaluating the proposed protocol with existing protocols.

1. Packet Loss: It is used to measure the strength of the protocol with respect to different node speed.

A protocol that receive less data loss as compared to other network protocol is considered as robust.

Blue Eyes Intelligence Engineering 
$\%$ of Packet loss $=$

Total number of packet lost

Total number of packettransmitted $X 100$

2. Packet delivery ratio: It provides an indication of the performance of a protocol to deliver packets to the respected destination.

$\%$ of Packet delivery ratio $=$

$\underline{\text { Total number of Packetdelivered successfuly }}$ X 100

Total number of packetsent by transmitter

3. Network Lifetime: It depends on the average energy consumption per round (work done) provided in the network. Lower the energy consumption of the node per round provides longer network lifetime.

It is defined as the number of round when first node dies in the network at a given instant of period.

\section{\% of Network Lifetime $=$}

$\frac{\text { Profit of time period }}{\text { Total number of time period } X \text { Number of nodes in the network }} \times 100$

4. Routing Overhead: It is the average control packets in which transmitting and receiving of packet consumes considerable amount of energy through neighboring nodes.In other words, Routing Overhead is measured as the amount of time taken to transmit the data packets from the source node to the sink node through the neighboring nodes. Routing Overhead =

(Number of data packets) $X$ (Time for routing of one data packet)

Example: Number of data packets are 10, time for routing one data packet is $1.1 \mathrm{~ms}$. Then the routing overhead is calculated as $11 \mathrm{~ms}$

5. Throughput: Rate of data sent from the cluster head to the base station [8,9]. And also the rate of data sent from member node to the cluster heads in the network.

$\%$ of Throughput $=$

$\frac{\text { (Number of successful packets) } X \text { (Average Packet size) }}{\text { Total time sent in delivering that amount of data }} X 100$

6. Latency: In any network topology, It is the total time that a data packet (Information source) to travel from one node to another node. when a data packet is transmitted and gets acknowledge to the source, the total time taken for for that one loop or round is known as latency[8, 9].

\section{Network Latency $=$}

$\left(\frac{\text { Distance fromone node to another node }}{\text { Transmission speed }}\right)+\left(\frac{\text { Packet size }}{\text { transmission rate }}\right)$

Energy model: When energy level of the node is less than the threshold value, then the free space model is used, otherwise multipath model is used.

Energy Consumption while transmission for path loss either free space or multipath fading is employed [YUAN ZHOU, (Senior Member), IEEE,March 13, 2017] [10].

Energy required to communicate $\mathrm{k}$ bit packets over a distance $\mathrm{d}$ is

$$
\begin{aligned}
& E_{T X}(k, d)=k \times E_{\text {elec }}+k \times E_{f s} \times d^{2}, \quad \text { if } d \leq d 0 \\
& k \times E_{\text {elec }}+k \times E_{\text {mp }} \times d^{4}, \quad \text { if } d>d 0
\end{aligned}
$$

The energy required to receive the $\mathrm{k}$ bit message is given by

$$
E_{R X}(k)=k \times E_{\text {elec. }}
$$

Optimum Number of Cluster: In Energy model relation, the optimum number of clusters uses a communication path for a given network protocol, where $\mathrm{N}$ number of nodes randomly distributed in a specific area is given by

$\mathrm{k}_{\mathrm{opt}}=\frac{\sqrt{N}}{\sqrt{2 \pi}} \sqrt{\frac{E f s}{E m p}} \frac{M}{{ }_{d_{\text {toBS }}}}$

To calculate the desired number of cluster in a specific network, the maximum and minimum values of distance from cluster head node to the base station $\mathrm{d}_{\mathrm{toBs}}$ should be applied to equation 4 . The interval $\mathrm{k}_{\mathrm{opt}}$ can be calculated based on the Quality of the network where, the number of packet received to the base station per time (Mehdi saeidmanesh, world academic of science, Engineering and technolgy,2009) [11].

Load Calculation: Load is calculated based on the number of node assigned earlier (confirmed) and the number of future possible assigned nodes. If the nodes are known earlier then the highest priority will be given compare to the newly assigned nodes.

Load of the node $=m P+n Q$

Where $P$ is the earlier nodes confirmed $Q$ is the newly arriving possible nodes $\mathrm{m}$ and $\mathrm{n}$ are the priorities of $\mathrm{P}$ and $\mathrm{Q}$.

\section{Parameters}

Few parameters to define cost function of the distributed cluster formation algorithm given in Table I.

Table- 1: Simulation parameter value

\begin{tabular}{|c|l|c|c|}
\hline $\begin{array}{r}\text { Sl } \\
\text { no. }\end{array}$ & \multicolumn{1}{|c|}{$\begin{array}{c}\text { Simulation } \\
\text { parameter }\end{array}$} & symbol & value \\
\hline 1 & Initial Energy & $\mathrm{E}_{0}$ & $\begin{array}{c}2 \mathrm{~J} \text { to } 6 \mathrm{~J} / \\
\text { Battery }\end{array}$ \\
\hline 2 & $\begin{array}{l}\text { Energy consumed } \\
\text { per bits of the } \\
\text { transmitter circuits }\end{array}$ & $\mathrm{E}_{\text {elec }}$ & $50 * 10^{-9} \mathrm{~J} / \mathrm{Bit}$ \\
\hline 3 & $\begin{array}{l}\text { Energy consumed } \\
\text { by the amplifier to } \\
\text { achieve an } \\
\text { acceptable SNR }\end{array}$ & $\mathrm{E}_{\mathrm{amp}}$ & $100 * 10^{-12} \mathrm{~J}$ \\
\hline 4 & $\begin{array}{l}\text { Energy used for } \\
\text { data gathering }\end{array}$ & $\mathrm{E}_{\mathrm{fs}}$ & $\begin{array}{c}10^{*} 10^{-12} \mathrm{~J} / \mathrm{Bit} / \\
\mathrm{m}^{2}\end{array}$ \\
\hline 5 & $\begin{array}{l}\text { Energy used for } \\
\text { multipath fading }\end{array}$ & $\mathrm{E}_{\mathrm{mp}}$ & $\begin{array}{c}0.003^{*} 10^{-12} \mathrm{~J} / \mathrm{B} \\
\mathrm{it} / \mathrm{m}^{4}\end{array}$ \\
\hline 6 & $\begin{array}{l}\text { Transmission } \\
\text { threshold distance }\end{array}$ & $\sqrt{\frac{\mathrm{d}_{0}}{\mathrm{E}_{\mathrm{s}}}}$ & \\
\hline
\end{tabular}

All the sensors are randomly distributed in a uniform area for the cluster formation for different networks.

1. Residual Energy of Cluster Head: Sensor nodes should be a member of cluster head which has higher residual energy than other node.

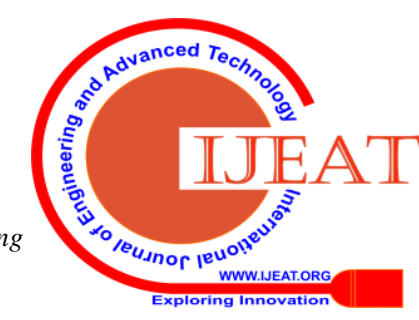


2. Distance from Cluster head to Base Station: The distance of the Cluster-head in each cluster network tends to be close to BS, which forces other cluster members to consume more energy to send data to the Cluster-head.

3. Distance from sensor node to Cluster-head: As the cluster members consume more energy for transmission to its cluster head. Sensor node should communicate the nearest cluster head. (Md Azharuddin,Elsevier ,2015)[12].

\section{SIMULATION RESULTS}

This article evaluates the performance of the proposed protocol through MATLAB Simulation. The Simulation parameters in Table II are based on the efficiency of the proposed protocol and compared with different protocols.

Table- II : proposed Simulation parameter value

\begin{tabular}{|c|l|c|}
\hline $\begin{array}{c}\text { Sl. } \\
\text { No. }\end{array}$ & Simulation parameter & $\begin{array}{c}\text { Proposed } \\
\text { values }\end{array}$ \\
\hline 1 & $\begin{array}{l}\text { Random node distribution } \\
\text { area }\end{array}$ & $\begin{array}{c}100 \mathrm{X} 100 \\
\text { And 150X200 }\end{array}$ \\
\hline 2 & Base station position placed & $\begin{array}{c}(0,150) \text { and } \\
(50,175)\end{array}$ \\
\hline 3 & Number of nodes & 100,200 \\
\hline 4 & The data packet size & 4000 bits \\
\hline 5 & Transmission range & 40 m \\
\hline 6 & MAC Layer & IEEE 802.11 \\
\hline 7 & Cluster head probability & 0.1 \\
\hline 8 & Control packet size & 200 bits \\
\hline 9 & Simulation time & 200 s \\
\hline 10 & $\begin{array}{l}\text { Total number of rounds } \\
\text { initiated }\end{array}$ & \\
\hline
\end{tabular}

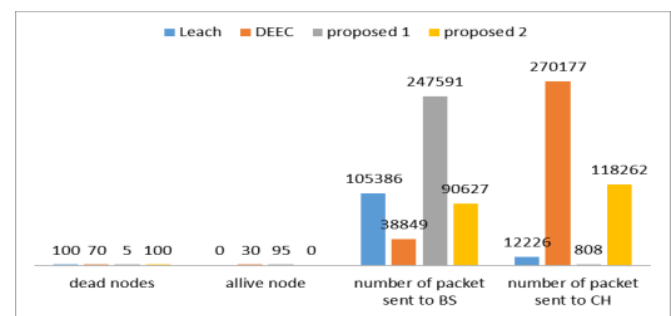

Fig 1: Simulation for 100 node with 5000 rounds for a Random node distribution area $100 \times 100$

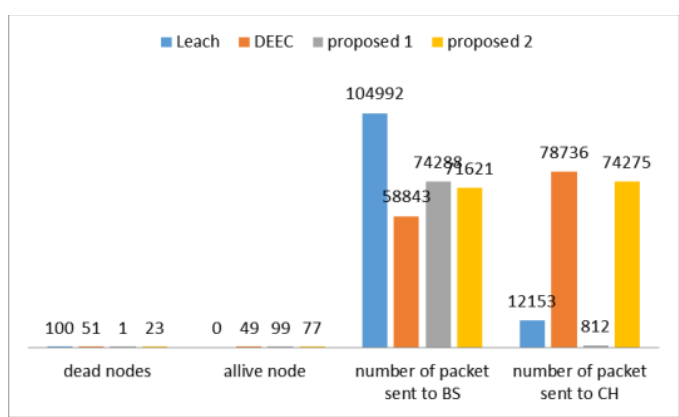

Fig 2: Simulation for 100 node with 5000 rounds for a Random node distribution area $100 \times 100$

Retrieval Number: E9946069520/2020@BEIESP DOI: 10.35940/ijeat.E9946.069520
Published By:

Blue Eyes Intelligence Engineering \& Sciences Publication

(C) Copyright: All rights reserved.

Fig 3: Simulation for 100 node with $\mathbf{5 0 0 0}$ rounds for a Random node distribution area 150X200

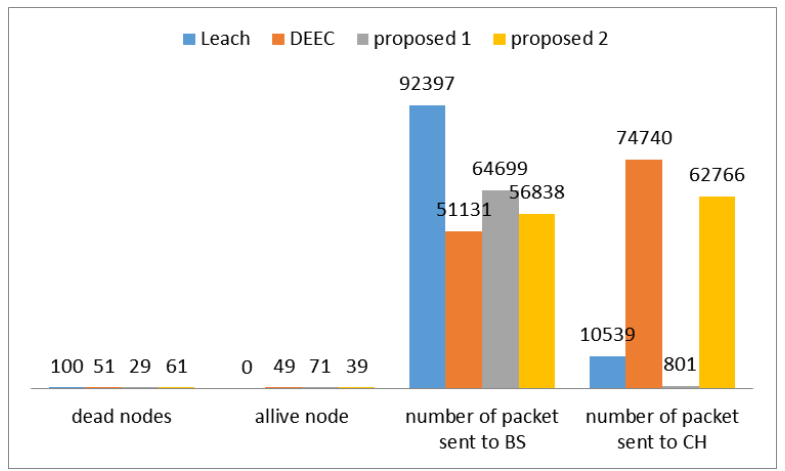

Fig 4: Simulation for 100 node with 1500 rounds for a Random node distribution area 150X200

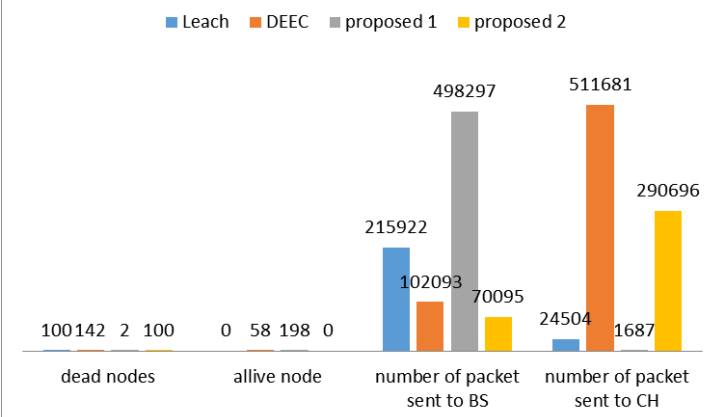

Fig 5: Simulation for 200 node with 5000 rounds for a Random node distribution area $150 \times 200$

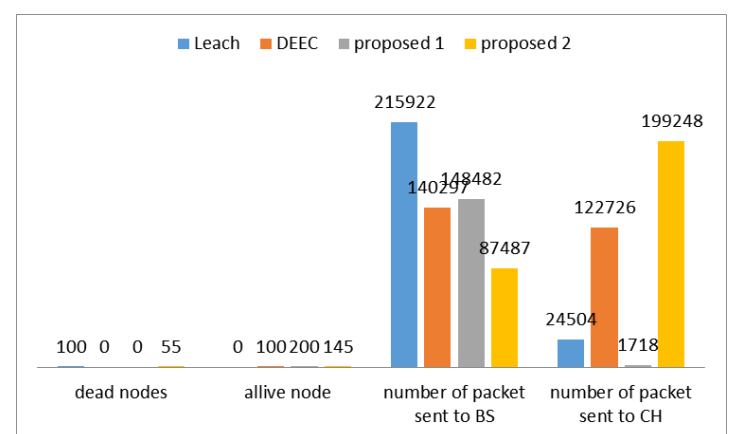

Fig 6: Simulation for 200 node with 1500 rounds for a Random node distribution area 100X100

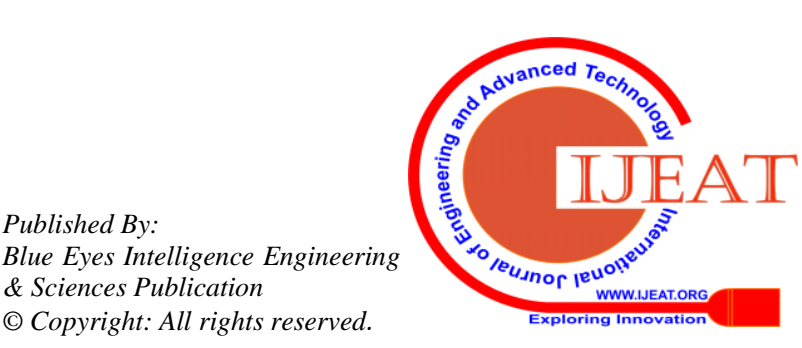




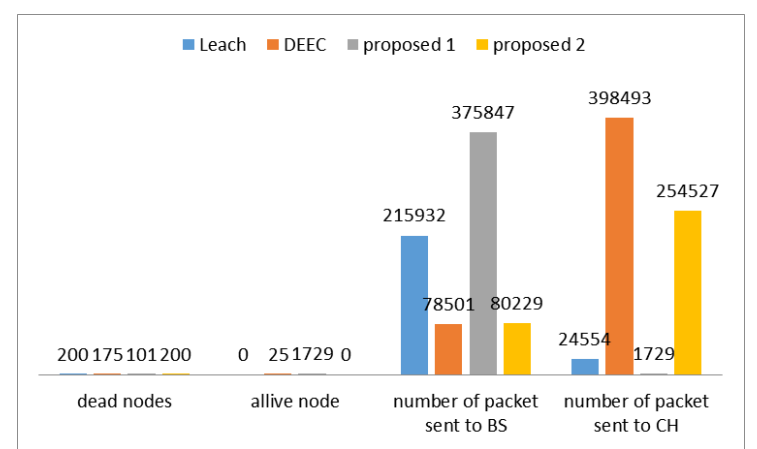

Fig 7: Simulation for 200 node with $\mathbf{5 0 0 0}$ rounds for a Random node distribution area 150X200

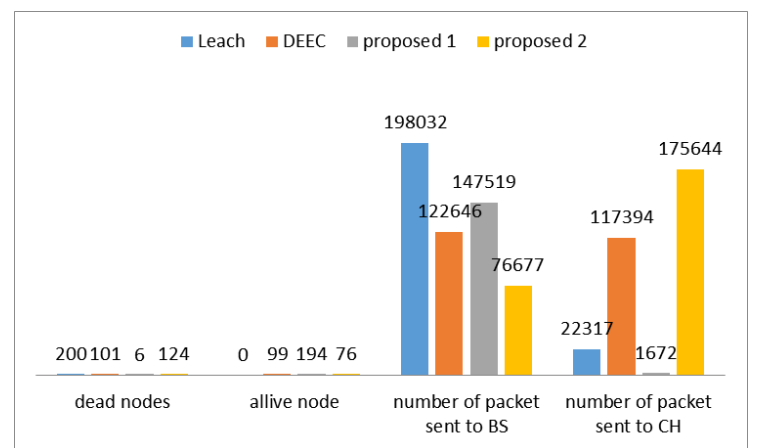

Fig 8: Simulation for 200 node with 1500 rounds for a Random node distribution area 150X200

\section{CONCLUSION}

This article investigates the efficiency of maximum lifetime cluster routing in a Random node distribution area. Creating a distributed frame network structure and minimizing the communication distance is to reduce the energy consumption of the network. The reviewed strategies commonly used to solve the wireless sensor network coverage problem and also the network lifetime to have their benefits and costs based on the prior knowledge of the network. Improving energy efficiency and minimize the total energy disbursed, balances the network lifetime. The performance of the network is influenced by these routing techniques. All these techniques have the common goal to increase the lifetime of the network.

\section{REFERENCES}

1. Li, Jian, and Prasant Mohapatra. "Analytical modeling and mitigation techniques for the energy hole problem in sensor networks." Pervasive and mobile Computing 3.3 (2007): 233-254.

2. Moro, Gianluca, and Gabriele Monti. "W-Grid: A scalable and efficient self-organizing infrastructure for multi-dimensional data management, querying and routing in wireless data-centric sensor networks." Journal of Network and Computer Applications 35.4 (2012): 1218-1234.

3. W. R. Heinzelman, A. Chandrakasan, and H. Balakrishnan, "Energy efficient communication protocol for wireless microsensor networks," in Proc. 33rd Annu. Hawaii Int. Conf. Syst. Sci., Jan. 2000, pp. 1-10.

4. Junping, Hu, Jin Yuhui, and Dou Liang. "A time-based cluster-head selection algorithm for LEACH." 2008 IEEE Symposium on Computers and Communications. IEEE, 2008

5. Bakaraniya, Parul, and Sheetal Mehta. "K-LEACH: An improved LEACH protocol for lifetime improvement in WSN." International Journal of Engineering Trends and Technology (IJETT) 4.5 (2013): 1521-1526.

6. Shukla, Indu, and N. Meghanathan. "Power Efficient Gathering in Sensor Information System (PEGASIS Protocol)." Jackson State University, Jackson MS, USA (2010).

7. Qing, Li, Qingxin Zhu, and Mingwen Wang. "Design of a distributed energy-efficient clustering algorithm for heterogeneous wireless sensor networks." Computer communications 29.12 (2006): 2230-2237.

Published By:

Blue Eyes Intelligence Engineering Publishing LLC, 2017. 177-190.
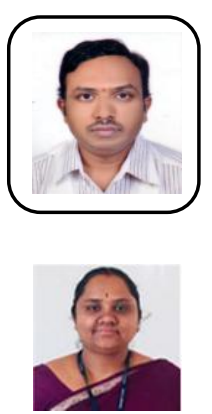

networks. Computing Systems (ICDCS'05). IEEE, 2005.

9. Ahmad, Afandi, Muhammad Faris Roslan, and Abbes Amira. "Throughput, latency and cost comparisons of microcontroller -based implementations of wireless sensor network (WSN) in high jump sports." AIP Conference Proceedings. Vol. 1883. No. 1. AIP

10. Zhou, Yuan, Ning Wang, and Wei Xiang. "Clustering hierarchy protocol in wireless sensor networks using an improved PSO algorithm." IEEE Access 5 (2016): 2241-2253.

11. Saeidmanesh, Mehdi, Mojtaba Hajimohammadi, and Ali Movaghar. "Energy and distance based clustering: An energy efficient clustering method for wireless sensor networks." World Academy of Science, Engineering and Technology 55 (2009): 555-559.

12. Azharuddin, Md, Pratyay Kuila, and Prasanta K. Jana. "Energy efficient fault tolerant clustering and routing algorithms for wireless sensor networks." Computers \& Electrical Engineering 41 (2015):

\section{AUTHORS PROFILE}

Harish S.V received his Post Graduate in 2010 from VTU, Belagavi. He is a Assistant professor in the Dept of Electronics and Communication Engineering, NIE Institute of Technology, Mysuru. His areas of research interest are Wireless sensor networks, communication protocols.

Dr. Archana NV, Received her doctorate in 2015 from University of Mysuru. She is a Professor and Head in the Dept of Electrical and Electronics Engineering, NIE Institute of Technology, Mysuru. Her area of research interests include adaptive control system, Wireless sensor

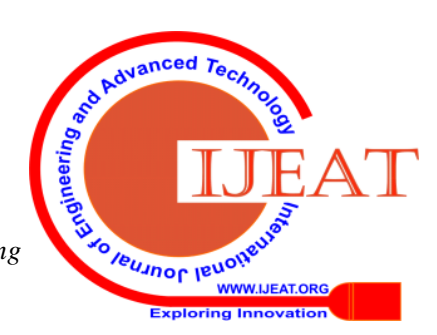

